\title{
VIOLATION OF BELL'S INEQUALITIES WITH A LOCAL THEORY OF PHOTONS
}

\author{
Patrick Suppes* J. Acacio de Barros ${ }^{\dagger}$ \\ Adonai S. Sant'Anna ${ }^{\ddagger}$ \\ Ventura Hall, Stanford University, \\ Stanford, California 94305-4115
}

August 24, 2021

\begin{abstract}
We use a local theory of photons purely as particles to model the single-photon experiment proposed by Tan, Walls, and Collett. Like Tan et al. we are able to derive a violation of Bell's inequalities for photon counts coincidence measurements. Our local probabilistic theory does not use any specific quantum mechanical calculations.

Key words: Bell's-inequalities, EPR, photons, hidden-variables, stochastic-models.
\end{abstract}

\section{Introduction}

The present paper is part of a research program which concerns a foundational analysis of phenomena usually described by quantum electrodynamics (QED) [6] [7] [8] [10]. Our previous papers give a particle theory for diffraction of light and the Casimir effect. The present paper is focused on another foundational topic. It remains to be seen how far the program we have undertaken can be carried.

\footnotetext{
*E-mail: suppes@ockham.stanford.edu. To whom correspondence should be addressed.

${ }^{\dagger}$ Permanent Address: Physics Department, Federal University at Juiz de Fora, 36036330 Juiz de Fora, MG Brazil. E-mail: acacio@fisica.ufjf.br

${ }^{\ddagger}$ Permanent Address: Mathematics Department, Federal University at Paraná, C.P. 19081, 81530-900, Curitiba, PR, Brazil. E-mail: adonai@gauss.mat.ufpr.br
} 
A probabilistic theory of photons with well-defined trajectories is assumed. The wave properties come from the expectation density of the photons. The photons are also regarded as virtual, because they are not directly observable, including their annihilation of each other (see assumptions bellow). What can be detected is the interaction with matter. The meaning of virtual used here is not the same as in QED. In summary, our basic assumptions are:

- Photons are emitted by harmonically oscillating sources;

- They have definite trajectories;

- They have a probability of being scattered by matter;

- Absorbers, like sources, are periodic;

- Photons have positive and negative states (+-photons and --photons) which locally interfere, when being absorbed.

The expected density of \pm -photons emitted at $t$ in the interval $d t$ is given by

$$
s_{ \pm}(t)=\frac{A_{s}}{2}(1 \pm \cos \omega t),
$$

where $\omega$ is the frequency of a harmonically oscillating source, $A_{s}$ is a constant determined by the source, and $t$ is time. We used $\frac{1}{2} \pm \frac{1}{2} \cos (\omega t)$ rather than $\cos (\omega t)$, to have a density that is nonnegative for all $t$ and is between 0 and 1. If a photon is emitted at $t^{\prime}, 0 \leq t^{\prime} \leq t$, then at time $t$ the photon has traveled (with speed $c$ ) a distance $r$, where

$$
t-t^{\prime}=\frac{r}{c}
$$

The conditional space-time expectation density of \pm -photons for a spherically symmetric source with given periodicity $\omega$ is:

$$
h_{ \pm}=\frac{A}{8 \pi r^{2}}\left(1 \pm \cos \omega\left(t-\frac{r}{c}\right)\right),
$$

where $A$ is a real constant.

The scalar field defined in terms of the expectation density $h_{ \pm}(t, r \mid \omega)$ is

$$
\mathcal{E}=\mathcal{E}_{0} \frac{h_{+}-h_{-}}{\sqrt{h_{+}+h_{-}}}
$$


where $\mathcal{E}_{0}$ is a scalar physical constant. Using (3), (41) may be rewritten for a spherically symmetric source as

$$
\mathcal{E}=\mathcal{E}_{0} \sqrt{\frac{A}{4 \pi r^{2}}} \cos \omega\left(t-\frac{r}{c}\right) .
$$

Applying the standard definition of average intensity, we get the expected result

$$
I=\left\langle\mathcal{E}^{2}\right\rangle=\frac{\mathcal{E}_{0}^{2} A}{8 \pi r^{2}}
$$

Note that the standard bracket notation is used for time averaging, i.e., taking an expectation with respect to $t$.

Since the absorber, or photodetector, behaves periodically with a frequency $\omega$, the probability $p_{X}$ of absorbing a photon in detector $X$ is given by

$$
p_{X}=\frac{C}{2}(1+\cos (\omega t+\psi))
$$

where $\psi$ is an arbitrary phase that can be randomized.

The expected number $E_{t}(X \pm)$ of each type of photon absorbed by detector $X$ is the time-averaged product

$$
E_{t}(X \pm)=\left\langle h_{ \pm}^{X}(\theta) p_{X}(\psi)\right\rangle
$$

where $h_{ \pm}^{X}(\theta)$ is the expected density of photons (with a phase $\theta$ ), and $\psi$ is a phase on detector $X$. Note that $E_{t}(X+)$, for example, is a random variable that is a function of $\theta$ and $\psi$. When we take expectation with respect to the distribution of $\theta$ we use subscripts to make clear that the expectation is with respect to $\theta$. The averaging is required because an absorption of an individual photon by an atom of a photodetector takes on average several orders of magnitude longer than the mean optical period of the photons, both theoretically and experimentally [4].

As we previously assumed, during the process of absorption, photons with different states (positive and negative) annihilate each other. So, the expected number of photons to be detected in each detector $X$ is:

$$
E_{t}(X)=\left|E_{t}(X+)-E_{t}(X-)\right| .
$$

We present here a violation of Bell's inequalities [1] [2] [3] with a local description of photons. 


\section{Experimental Configuration}

We are interested in the experimental setup proposed in 11] and also discussed in [12]. The scheme uses two coherent sources $\alpha_{1}\left(\theta_{1}\right)$, with phase $\theta_{1}$, and $\alpha_{2}\left(\theta_{2}\right)$, with phase $\theta_{2}$, and a third source to be studied, $u(\theta)$, with unknown phase. The experimental configuration has two homodyne detections, $\left(D_{1}, D_{2}\right)$ being one and $\left(D_{3}, D_{4}\right)$ the other, such that the measurements are sensitive to phase changes in $u(\theta)$. The geometry of the setup is shown in Figure 1. In Figure 1 BS1, BS2 and BS3 are beam splitter mirrors that will

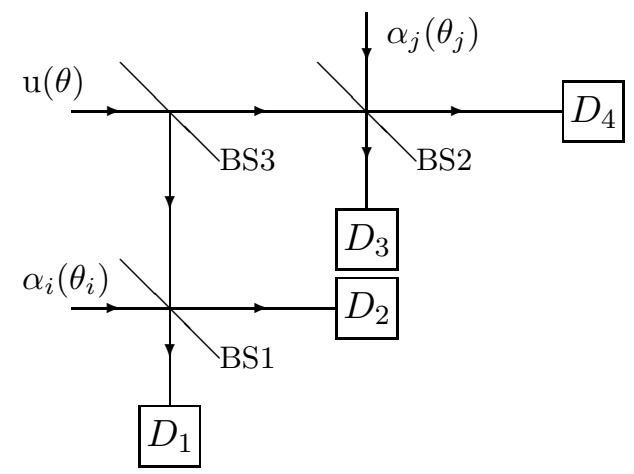

Figure 1: Proposed experimental configuration.

reflect $50 \%$ of the incident photons and let $50 \%$ of them pass. When photons are reflected, the mirrors add a phase of $\pi / 2$ to the expected density, while no phase is added to the expected density when photons pass through $\mathrm{BS} 1, \mathrm{BS} 2$ or BS3. It is easy to devise a way to have the expected density of photons changed by a $\pi / 2$ phase by just delaying the photons that are reflected, and hence have interacted with the mirror, by a time $T / 4$, where $T$ is the period of the photon source. We will look for correlations between the pairs of photon detectors $\left(D_{1}, D_{2}\right)$ and $\left(D_{3}, D_{4}\right)$.

The expected density of \pm -photons, generated by the source $u(\theta)$ is:

$$
h_{ \pm}^{u}(\theta)=\frac{\beta}{2}(1 \pm \cos (\omega t+\theta))
$$

The expected density coming from $u(\theta)$ at each detector is:

$$
h_{ \pm}^{D_{1}}(\theta)=\frac{\beta}{8}\left(1 \pm \cos \left(\omega t+\theta+\frac{\pi}{2}\right)\right)
$$




$$
\begin{gathered}
h_{ \pm}^{D_{2}}(\theta)=\frac{\beta}{8}(1 \pm \cos (\omega t+\theta+\pi)), \\
h_{ \pm}^{D_{3}}(\theta)=\frac{\beta}{8}\left(1 \pm \cos \left(\omega t+\theta+\frac{\pi}{2}\right)\right), \\
h_{ \pm}^{D_{4}}(\theta)=\frac{\beta}{8}(1 \pm \cos (\omega t+\theta)) .
\end{gathered}
$$

Note that we neglected factors of the form $\mathbf{k} \cdot \mathbf{x}$ coming from path contributions to the phase. We can do so considering the problem completely symmetric and remembering that only phase differences are relevant for the measurements we are proposing.

In similar fashion, the expected density of \pm -photons generated by the coherent sources $\alpha_{i}$, with phase $\theta_{i}$ and amplitude $\alpha / 2$, and $\alpha_{j}$, with phase $\theta_{j}$ and amplitude $\alpha / 2$, in each detector, is given by the following expressions:

$$
\begin{gathered}
h_{ \pm}^{D_{1}}\left(\theta_{i}\right)=\frac{\alpha}{4}\left(1 \pm \cos \left(\omega t+\theta_{i}+\frac{\pi}{2}\right)\right), \\
h_{ \pm}^{D_{2}}\left(\theta_{i}\right)=\frac{\alpha}{4}\left(1 \pm \cos \left(\omega t+\theta_{i}\right)\right), \\
h_{ \pm}^{D_{3}}\left(\theta_{j}\right)=\frac{\alpha}{4}\left(1 \pm \cos \left(\omega t+\theta_{j}\right)\right), \\
h_{ \pm}^{D_{4}}\left(\theta_{j}\right)=\frac{\alpha}{4}\left(1 \pm \cos \left(\omega t+\theta_{j}+\frac{\pi}{2}\right)\right),
\end{gathered}
$$

where we again neglected path contributions to the phase and considered only the relevant phase at the detectors.

We should point out that in equations (10)-(18) $\alpha$ and $\beta$ are split in half at each semi-mirror, because each time a photon reaches a mirror there is a probability of $1 / 2$ that the photon passes through and a probability of $1 / 2$ that the photon is reflected by the mirror.

The probability of absorption in each detector, consistent with equation (7), is given most simply by the following equations. Some alternatives are formulated in equations (40)-(47) at the end of this section.

$$
\begin{gathered}
p_{D_{1}}=\frac{C}{4}\left(2+\cos \left(\omega t+\theta_{i}+\frac{\pi}{2}\right)+\cos \left(\omega t+\theta+\frac{\pi}{2}\right)\right), \\
p_{D_{2}}=\frac{C}{4}\left(2+\cos \left(\omega t+\theta_{i}+\pi\right)+\cos (\omega t+\theta)\right), \\
p_{D_{3}}=\frac{C}{4}\left(2+\cos \left(\omega t+\theta_{j}\right)+\cos \left(\omega t+\theta+\frac{\pi}{2}\right)\right),
\end{gathered}
$$




$$
p_{D_{4}}=\frac{C}{4}\left(2+\cos \left(\omega t+\theta_{j}+\frac{\pi}{2}\right)+\cos (\omega t+\theta)\right),
$$

where $C$ is a constant that corresponds to the efficiency of the detection process.

The expected number of \pm photons in each detector is given, according to equation (8), by the following expressions:

$$
\begin{aligned}
& E_{t}\left(D_{1}^{ \pm}\right)=\left\langle\left(h_{ \pm}^{D_{1}}\left(\theta_{i}\right)+h_{ \pm}^{D_{1}}(\theta)\right) p_{D_{1}}\right\rangle, \\
& E_{t}\left(D_{2}^{ \pm}\right)=\left\langle\left(h_{ \pm}^{D_{2}}\left(\theta_{i}\right)+h_{ \pm}^{D_{2}}(\theta)\right) p_{D_{2}}\right\rangle, \\
& E_{t}\left(D_{3}^{ \pm}\right)=\left\langle\left(h_{ \pm}^{D_{3}}\left(\theta_{j}\right)+h_{ \pm}^{D_{3}}(\theta)\right) p_{D_{3}}\right\rangle, \\
& E_{t}\left(D_{4}^{ \pm}\right)=\left\langle\left(h_{ \pm}^{D_{4}}\left(\theta_{j}\right)+h_{ \pm}^{D_{4}}(\theta)\right) p_{D_{4}}\right\rangle .
\end{aligned}
$$

Equations (23) - 26) use the fact that the expected number of photons at a detector is simply the sum of the number of photons from all sources. Also, in the equations above $\langle F(t)\rangle=\frac{1}{T} \int_{0}^{T} F(t) d t$, represents a time average of the random variable $F(t)$, where $t$ is time and $(0, T)$ is a time interval such that $\omega T \gg 1$. It is straightforward to obtain the expressions for the total expected number of photons in each detector from equations (3), (9), (15) (18), (19) -(22), and (23)-(26), which we write as $I_{k}$, for $k=1, \ldots, 4$, with $I_{k}$ a function of $\theta$ and $\theta_{i}$ or $\theta_{j}$ :

$$
\begin{aligned}
& I_{1}=\left|E_{t}\left(D_{1}^{+}\right)-E_{t}\left(D_{1}^{-}\right)\right|=\frac{C}{16}\left|\alpha+\frac{\beta}{2}+\left(\alpha+\frac{1}{2} \beta\right) \cos \left(\theta-\theta_{i}\right)\right|, \\
& I_{2}=\left|E_{t}\left(D_{2}^{+}\right)-E_{t}\left(D_{2}^{-}\right)\right|=\frac{C}{16}\left|\alpha+\frac{\beta}{2}-\left(\alpha+\frac{1}{2} \beta\right) \cos \left(\theta-\theta_{i}\right)\right|, \\
& I_{3}=\left|E_{t}\left(D_{3}^{+}\right)-E_{t}\left(D_{3}^{-}\right)\right|=\frac{C}{16}\left|\alpha+\frac{\beta}{2}-\left(\alpha+\frac{1}{2} \beta\right) \sin \left(\theta-\theta_{j}\right)\right|, \\
& I_{4}=\left|E_{t}\left(D_{4}^{+}\right)-E_{t}\left(D_{4}^{-}\right)\right|=\frac{C}{16}\left|\alpha+\frac{\beta}{2}+\left(\alpha+\frac{1}{2} \beta\right) \sin \left(\theta-\theta_{j}\right)\right| .
\end{aligned}
$$

The expressions on the right hand side of (27) - (30) are nonnegative, independent of taking their absolute value, and so we subsequently drop the absolute values.

We are interested in the correlation between the two pairs of detectors. First we need the variances

$$
\operatorname{Var}_{\theta}\left(I_{1}-I_{2}\right)=E_{\theta}\left(\left(I_{1}-I_{2}\right)^{2}\right)-\left(E_{\theta}\left(I_{1}-I_{2}\right)\right)^{2},
$$




$$
\operatorname{Var}_{\theta}\left(I_{3}-I_{4}\right)=E_{\theta}\left(\left(I_{3}-I_{4}\right)^{2}\right)-\left(E_{\theta}\left(I_{3}-I_{4}\right)\right)^{2},
$$

and covariance

$$
\operatorname{Cov}_{\theta}\left(\left(I_{1}-I_{2}\right)\left(I_{3}-I_{4}\right)\right)=E_{\theta}\left(\left(I_{1}-I_{2}\right)\left(I_{3}-I_{4}\right)\right)-E_{\theta}\left(I_{1}-I_{2}\right) E_{\theta}\left(I_{3}-I_{4}\right),
$$

where $E_{\theta}\left(I_{k}\right)=\frac{1}{2 \pi} \int_{0}^{2 \pi} I_{k} d \theta$, for $k=1, \ldots, 4$, is an expectation with respect to $\theta$, with $\theta$ uniformly distributed on $[0,2 \pi]$. Thus

$$
\begin{aligned}
& \operatorname{Var}_{\theta}\left(I_{1}-I_{2}\right)=\frac{1}{512} C^{2}(\beta+2 \alpha)^{2}, \\
& \operatorname{Var}_{\theta}\left(I_{4}-I_{3}\right)=\frac{1}{512} C^{2}(\beta+2 \alpha)^{2},
\end{aligned}
$$

and

$$
\operatorname{Cov}_{\theta}\left(\left(I_{1}-I_{2}\right)\left(I_{3}-I_{4}\right)\right)=-\frac{1}{512} C^{2}(\beta+2 \alpha)^{2} \sin \left(\theta_{i}-\theta_{j}\right) .
$$

The correlation is given by

$$
\rho_{\theta}\left(I_{1}-I_{2}, I_{3}-I_{4}\right)=\frac{\operatorname{Cov}_{\theta}\left(\left(I_{1}-I_{2}\right)\left(I_{3}-I_{4}\right)\right)}{\sqrt{\operatorname{Var}_{\theta}\left(I_{1}-I_{2}\right) \operatorname{Var}_{\theta}\left(I_{3}-I_{4}\right)}},
$$

or

$$
\rho_{\theta}\left(I_{1}-I_{2}, I_{3}-I_{4}\right)=-\sin \left(\theta_{i}-\theta_{j}\right) .
$$

It is easy to show that (38) violates Bell's inequalities when four appropriate phases are chosen.

An examination of the derivation of (38) shows that without serious change it holds simply for a classical field as (5). Details and discussion can be found in [9]. In the case of both $\operatorname{Var}_{\theta}$ and $\operatorname{Cov}_{\theta}$, it is important to note that if we computed the correlation with respect to $t$ rather than $\theta$, we would get different results. It is easy to show, for example, that

$$
\operatorname{Var}_{\theta} E_{t}\left(D_{1}^{ \pm}\right) \neq \operatorname{Var}_{t} E_{\theta}\left(D_{1}^{ \pm}\right)
$$

In contrast, the order of $\theta$ and $t$ does not matter in analyzing the data of discrete photon counts, in Section 3.

An attentive reader may object to our expression for the probability of detection, because we assume that the detector has the same probability to oscillate in phase with the noncoherent source as it has to oscillate with the coherent source, and that may bring some non-local characteristics to the 
model. We can respond to this by examining the following probability for absorption.

$$
\begin{gathered}
p_{D_{1}}=\frac{C}{2}\left(1+\cos \left(\omega t+\theta_{i}+\frac{\pi}{2}\right)\right), \\
p_{D_{2}}=\frac{C}{2}\left(1+\cos \left(\omega t+\theta_{i}\right)\right), \\
p_{D_{3}}=\frac{C}{2}\left(1+\cos \left(\omega t+\theta_{j}\right)\right), \\
p_{D_{4}}=\frac{C}{2}\left(1+\cos \left(\omega t+\theta_{j}+\frac{\pi}{2}\right)\right) .
\end{gathered}
$$

The probabilities above have no term on $\theta$, but depend only on the phase of the coherent sources. This fact have the effect of wiping out all influences that the non-coherent source have on the detectors, and hence putting only local parameters, like $\theta_{i}$ or $\theta_{j}$, depending on the detector, in the probability for detection. If we redo the computations for the correlation with the probabilities above, we end up with the same correlation function for a pair of homodyne detections. In fact, to point out the robustness of the result in face of the choice of probability for detection, we may examine the following set of probabilities.

$$
\begin{gathered}
p_{D_{1}}=\frac{C}{2}\left(1+\frac{\alpha \cos \left(\omega t+\theta_{i}+\frac{\pi}{2}\right)+\beta \cos \left(\omega t+\theta+\frac{\pi}{2}\right)}{\alpha+\beta}\right), \\
p_{D_{2}}=\frac{C}{2}\left(1+\frac{\alpha \cos \left(\omega t+\theta_{i}+\pi\right)+\beta \cos (\omega t+\theta)}{\alpha+\beta}\right) \\
p_{D_{3}}=\frac{C}{2}\left(1+\frac{\alpha \cos \left(\omega t+\theta_{j}\right)+\beta \cos \left(\omega t+\theta+\frac{\pi}{2}\right)}{\alpha+\beta}\right) \\
p_{D_{4}}=\frac{C}{2}\left(1+\frac{\alpha \cos \left(\omega t+\theta_{j}+\frac{\pi}{2}\right)+\beta \cos (\omega t+\theta)}{\alpha+\beta}\right) .
\end{gathered}
$$

The above expressions would have a different physical interpretation from the previous two presented. Each phase is given a probability that is proportional to the amplitude of the source with the corresponding phase. The stronger the source, the more probable to find the detector with the same phase. It is again easy to show that if we use these probabilities we get the same correlations as before. 


\section{Photon Counts that Violate Bell's Inequalities}

In this section we are going to use the previous result to model discrete photon counts in such a way that they violate Bell's inequalities. For this, we define two new discrete random variables $X= \pm 1$ and $Y= \pm 1$. These random variables correspond to nearly simultaneous correlated counts at the detectors, and are defined in the following way.

$$
\begin{aligned}
& X= \begin{cases}+1 & \text { if a photon is detected at } D_{1} \\
-1 & \text { if a photon is detected at } D_{2}\end{cases} \\
& Y= \begin{cases}+1 & \text { if a photon is detected at } D_{3} \\
-1 & \text { if a photon is detected at } D_{4}\end{cases}
\end{aligned}
$$

To compute the expectation of $X$ and $Y$ we use the stationarity of the process and do the following. First, let us note that

$$
I_{1}-I_{2}=N_{X} \cdot P(X=1)-N_{X} \cdot P(X=-1),
$$

where $N_{X}$ is the expected total number of photons detected at $D_{1}$ and $D_{2}$ and $P(X= \pm 1)$ is the probability that the random variable $X$ has values \pm 1 . The same relation holds for

$$
I_{3}-I_{4}=N_{Y} \cdot P(Y=1)-N_{Y} \cdot P(Y=-1) .
$$

To simplify we put as a symmetry condition that $N_{X}=N_{Y}=N$, i.e., the expected number of photons hitting each homodyne detector is the same. But we know that

$$
I_{1}+I_{2}=N \cdot P(X=1)+N \cdot P(X=-1)=N,
$$

and

$$
I_{3}+I_{4}=N \cdot P(X=1)+N \cdot P(X=-1)=N .
$$

Then we can conclude from equations (27)- 30 ) that

$$
\begin{aligned}
& E_{d}(X \mid \theta)=\frac{I_{1}-I_{2}}{I_{1}+I_{2}}=\cos \left(\theta-\theta_{i}\right), \\
& E_{d}(Y \mid \theta)=\frac{I_{3}-I_{4}}{I_{3}+I_{4}}=\sin \left(\theta-\theta_{j}\right),
\end{aligned}
$$


where $E_{d}$ represents the expected value of the counting random variable. It is clear that if $\theta$ is uniformly distributed we have at once:

$$
\begin{aligned}
& E(X)=E_{\theta}\left(E_{d}(X \mid \theta)\right)=0, \\
& E(Y)=E_{\theta}\left(E_{d}(X \mid \theta)\right)=0 .
\end{aligned}
$$

We can now compute $\operatorname{Cov}(X, Y)$. Note that

$$
\begin{aligned}
\operatorname{Cov}(X, Y) & =E(X Y)-E(X) E(Y) \\
& =E_{\theta}\left(E_{d}(X Y \mid \theta)\right)-E_{\theta}\left(E_{d}(X \mid \theta)\right) E_{\theta}\left(E_{d}(Y \mid \theta)\right)
\end{aligned}
$$

and so

$$
\begin{aligned}
\operatorname{Cov}(X, Y)= & \frac{1}{2 \pi} \int_{0}^{2 \pi} E_{d}(X Y \mid \theta) d \theta \\
& -\frac{1}{2 \pi} \int_{0}^{2 \pi} E_{d}(X \mid \theta) d \theta \times \frac{1}{2 \pi} \int_{0}^{2 \pi} E_{d}(Y \mid \theta) d \theta .
\end{aligned}
$$

In order to compute the covariance, we also use the conditional independence of $X$ and $Y$ given $\theta$, which is our locality condition:

$$
E_{d}(X Y \mid \theta)=E_{d}(X \mid \theta) E_{d}(Y \mid \theta)
$$

because given $\theta$, the expectation of $X$ depends only on $\theta_{i}$, and of $Y$ only on $\theta_{j}$. Then, it is easy to see that

$$
\rho(X, Y)=\operatorname{Cov}(X, Y)=-\sin \left(\theta_{i}-\theta_{j}\right)
$$

The correlation equals the covariance, since $X$ and $Y$ are discrete \pm 1 random variables with zero mean, as shown in (56) and (57), and so $\operatorname{Var}(X)=$ $\operatorname{Var}(Y)=1$. It follows at once from (61) that for a given set of $\theta_{i}$ 's and $\theta_{j}$ 's Bell's inequalities are violated.

\section{Acknowledgments}

J. A. B. acknowledges support from the Department of Fields and Particles (DCP) of the Brazilian Center for Physics Research (CBPF/CNPq). A. S. S. acknowledges financial support from CNPq (Brazilian Government's Support Agency). 


\section{References}

[1] Bell, J. S., "On the Einstein Podolsky Rosen Paradox," Physics 1195 (1964).

[2] Bell, J. S., "On the Problem of Hidden Variables in Quantum Mechanics," Reviews of Modern Physics 38447 (1966).

[3] Clauser, J. F., and A. Shimony, "Bell's Theorem: Experimental Tests and Implications," Rep. Prog. Phys. 41, 1881 (1978).

[4] Nussenzveig, H. M., Introduction to Quantum Optics (Gordon \& Breach, N.Y., 1973).

[5] Suppes, P. and M. Zanotti, "On the Determinism of Hidden Variable Theories with Strict Correlation and Conditional Statistical Independence of Observables," in Logic and Probability in Quantum Mechanics, edited by P. Suppes, D. Reidel Publishing Company, 445 (1976).

[6] Suppes, P. and J. A. de Barros, "A Random-Walk Approach to Interference," Int. J. of Theor. Phys. 33, 179 (1994a).

[7] Suppes, P. and J. A. de Barros, "Diffraction With Well-Defined Photon Trajectories: a Foundational Analysis," Found. Phys. Lett. 7, 501 (1994b).

[8] Suppes, P. and J. A. de Barros, "Photons, Billiards and Chaos," In Press (1996).

[9] Suppes, P., J. A. de Barros, and A. S. Sant'Anna, "A Proposed Experiment Showing that Classical Fields Can Violate Bell's Inequalities," to appear.

[10] Suppes, P., A. S. Sant'Anna, and J. A. de Barros, "A Particle Theory of the Casimir Effect," submitted to Found. Phys. Lett.

[11] Tan, S. M., D. F. Walls, and M. J. Collett, "Nonlocality of a Single Photon," Phys. Rev. Lett., 66 252-255 (1991).

[12] Walls, D. F. and G. J. Milburn, Quantum Optics, Springer-Verlag (1994). 